\title{
Effect of Low Molecular Weight Fucoidan and Low Molecular Weight Heparin in a Rabbit Model of Arterial Thrombosis
}

\author{
Eric Durand ${ }^{\mathrm{a}, \mathrm{c},{ }^{*}}$, Dominique Helley ${ }^{\mathrm{a}, \mathrm{c}, \mathrm{d}}$, Ayman Al Haj Zen ${ }^{\mathrm{a}, \mathrm{b}}$, Céline Dujols ${ }^{\mathrm{d}}$, Patrick \\ Bruneval $^{a, c, e}$, Sylvia Colliec-Jouault ${ }^{f}$, Anne-Marie Fischer ${ }^{a, c, d}$, Antoine Lafont ${ }^{a, c}$
}

\footnotetext{
${ }^{a}$ Faculté de Médecine, Université Paris Descartes,

b INSERM Unité 849,

c Assistance Publique-Hôpitaux de Paris, Hôpital Européen Georges Pompidou,

dINSERM Unité 765, and

e INSERM Unité 430, Paris, and

${ }^{f}$ IFREMER, Laboratoire Biotechnologie et Molécules Marines, Nantes, France
}

*: Corresponding author : E. Durand, Tel. +33 15609 3692, Fax +33 15609 2664, email address : eric.durand@egp.aphp.fr

\begin{abstract}
:
Background: Therapeutic use of unfractionated heparin and low molecular weight heparins (LMWHs) is limited by hemorrhagic adverse effects. We compared the antithrombotic effect of LMW fucoidan (LMWF) and LMWH in an experimental model. Methods: Thrombosis was induced in femoral arteries of male New Zealand White rabbits by in situ induction of endothelial apoptosis with staurosporine $\left(10^{-}\right.$ ${ }^{5} \mathrm{M}$ for $\left.30 \mathrm{~min}\right)$. Starting the day before apoptosis induction, the animals received subcutaneous LMWF (15 mg/kg), LMWH (enoxaparin $2.5 \mathrm{mg} / \mathrm{kg}$ ) or saline solution (control group) twice a day for 4 days. Results: The degrees of apoptosis and endothelial denudation were similar in the 3 groups. The thrombotic score was significantly lower in the LMWF group than in the LMWH and control groups ( $p=$ 0.01). Tissue factor expression was significantly lower in the LMWF group than in the control and LMWH groups $(p=0.01)$. The plasma concentration of tissue factor pathway inhibitor was significantly increased after LMWF injection (137 \pm 28 vs. $102 \pm 17 ; p=0.01)$, whereas no change was observed after LMWH treatment. LMWF did not prolong the bleeding time or decrease platelet aggregation. Conclusions: LMWF appeared to be more effective than LMWH for preventing arterial thrombosis in this experimental model. LMWF also had a lower hemorrhagic risk than LMWH.
\end{abstract}

Keywords: Thrombosis, Fucoidan, Heparin, Tissue factor pathway inhibitor, Tissue factor 


\section{Introduction}

Unfractionated (UF) or low molecular weight heparins (LMWHs) are commonly used to reduce thrombin formation in association with antiplatelet therapy in arterial thrombotic diseases. However, the antithrombotic effect of UF and LMWHs is limited by their anticoagulant activity and thus their hemorrhagic adverse effects. Changer la phrase en disant plus clairement qu'en augmentant la dose on améliore leur effet antithrombotique mais également les effets indésirables hémorragiques. Fucoidan, a sulfated polysaccharide extracted from brown seaweeds, has anticoagulant and antithrombotic effects by catalyzing thrombin inhibition mainly by heparin cofactor II, according to its chemical structure [1, 2]. Moreover, as previously described for heparins [3, 4], fucoidan induces tissue factor pathway inhibitor (TFPI) release from cultured human umbilical vein endothelial cells which may also contribute to its antithrombotic effect [5]. A LMW fucoidan (LMWF) of $7 \mathrm{kDa}$ can be obtained by chemical degradation [1]. The LMWF has been previously compared to LMWH. When used at the same venous antithrombotic activity, LMWF did not induce a prolongation of the thrombin clotting time (TCT) or an increase in anti-Xa activity, contrary to LMWH, which corresponds to a lower hemorrhagic risk. A slight prolongation of activated partial thromboplastin time (APTT) was however observed with both compounds [6, 7]. We have recently developed a model of arterial thrombosis by local stimulation of endothelial apoptosis with staurosporin associated with endothelial denudation reproducing the two major components of plaque erosion in humans [8]. We therefore aimed to evaluate the antithrombotic and the anticoagulant effects of LMWF and LMWH in this experimental model of arterial thrombosis mimicking a pathological situation in human.

\section{Material and methods}

\subsection{Materials}

LMWH [enoxaparin: molecular mass 5,000 g/mol; anticoagulant activity: 10000 anti-Xa IU/ml (100 mg/mL)] was obtained from Sanofi Aventis (France).

LMWF was obtained from IFREMER (Nantes, France) by a radical depolymerization process, as previously described [1].

\subsection{Experimental model of arterial thrombosis}

The investigation conforms to previously published guidelines by the US National Institutes of Health, and were approved by our local animal experiment committee. Arterial thrombosis was induced in male New Zealand White rabbit femoral arteries by in situ induction of endothelial apoptosis by staurosporin, as previously described [8]. Male New Zealand White rabbits $(n=18), 3-4 \mathrm{~kg}$, were anesthetized with the combination of Xylazine $(5 \mathrm{mg} / \mathrm{Kg})$ and Ketamine $(35 \mathrm{mg} / \mathrm{Kg})$. In each femoral artery, ligatures were used to isolate a segment ( $1 \mathrm{~cm}$ long) that was punctured proximally by a 27 Gauge needle and incubated with staurosporin $\left(10^{-5} \mathrm{M}\right)$ in each artery. After 30 min-incubation, femoral segments were washed twice with saline and re-exposed to the circulating blood flow.

\subsection{Therapeutic design}

Three groups of animals underwent the surgical procedure. In the first group (control group, $n=6$ ), animals received twice daily a subcutaneous injection of a saline solution beginning 1 day before the surgical procedure until the sacrifice. In the second and third groups ( $n=6$ each), animals received similarly LMWF or LMWH. The design of this preclinical study was defined by both safety and efficacy concerns. Thus, a doseeffect curve was performed with $\operatorname{LMWF}(7.5,10$, and $15 \mathrm{mg} / \mathrm{kg})$ and $\operatorname{LMWH}(1,2.5,5,10$, and $15 \mathrm{mg} / \mathrm{kg}) \mathrm{in}$ order to determine the optimal dose of both polysaccharides for the in vivo antithrombotic study. Four animals were used for each dose. APTT, thrombin clotting time (TCT), and anti-Xa activity were analysed one hour after subcutaneous injection of LMW polysaccharides. TCT and anti-Xa activity were not significantly altered with LMWF. APTT slightly increased (twice fold) at $15 \mathrm{mg} / \mathrm{Kg}$. APTT, TCT, and anti-Xa activity were very high with LMWH from 5 to $15 \mathrm{mg} / \mathrm{kg}$, and should induce in vivo hemorrhagic adverse effects. Moreover, at $2.5 \mathrm{mg} / \mathrm{kg}$, LMWH induced a twice-fold increase of APTT similar as those obtained with LMWF. We therefore chose the dose of LMW polysaccharides inducing the same doubling of APTT (i.e, 2.5 and $15 \mathrm{mg} / \mathrm{kg}$ for LMWH and LMWF, respectively). 


\subsection{Assessment of vessel thrombosis and immunochemistry}

Thrombosis occurrence was evaluated by angiography and histology 3 days after induction of endothelial apoptosis. Thrombus was defined by histology as an adherent intra luminal material responsible of partial or total lumen occlusion, as previously described [8]. A thrombotic score was used to evaluate the severity of the thrombotic response, as follows; 0: no thrombosis; 1 : thrombus less than $50 \%$ of lumen vessel obstruction; 2: between $50 \%$ and $75 \%$; and 3: thrombus more than $75 \%$.

In order to evaluate endothelial denudation, femoral arteries sections were stained with a monoclonal antiCD31 antibody (Dako, Trappes, France). In situ detection of apoptotic cells was performed using TUNEL (Apoptag kit, Oncor, Gaithesburg, MD). A semi quantitative apoptotic score evaluating the extent of apoptosis in the endothelial lining was established by a blinded observer, as follows: 0 indicated no or barely detectable staining; 1 , weak positive staining; 2 , moderate limited staining; and 3 , strong diffuse staining [8]. Areas where endothelial cell lining was absent (negative CD31 staining) were considered as resulting from apoptosis-induced endothelial denudation, and were counted as apoptotic (score 3). Finally, we quantified the percentage of endothelial cells (total and apoptotic) remaining after staurosporin treatment, as previously described [8].

Sections were stained with a monoclonal anti-rabbit tissue factor (TF) antibody (American Diagnostica, Greenwich, CT) in order to evaluate TF expression. A semi quantitative score was used to detect TF expression by a blinded observer, as follows: 0 indicated no or barely detectable staining; 1 , weak positive staining; 2, moderate limited staining; and 3, strong diffuse staining, as previously described [9].

\subsection{Measurements of anticoagulant activity}

Three blood samples from each animal were collected in 3.8\% sodium citrate in order to determine ex vivo anticoagulant activity at baseline, 1 hour after the second subcutaneous injection; and 1 hour after the final injection (the $8^{\text {th }}$ injection). Platelet-poor plasma was obtained by two centrifugations at $1000 \mathrm{~g}$ for $10 \mathrm{~min}$ at $20^{\circ} \mathrm{C}$ and stored at $-80^{\circ} \mathrm{C}$ until use.

Ex vivo anticoagulant activities were evaluated on an STAR coagulation analyser (Stago, Gennevilliers, France) by an activated partial thromboplastin time (Automated APTT, BioMerieux, Durham, NC), a thrombin clotting time (TCT, Thrombin Sigma Aldrich, Saint-Louis, MO), and an anti-Xa activity with commercial chromogenic assay (STA Rotachrom Heparin 8, Stago, Gennevilliers, France) in the 3 groups of animals.

\subsection{Measurements of bleeding time and platelet aggregation}

Bleeding time and platelet aggregation were evaluated 1 hour after the final injection in each group. The bleeding time (expressed in sec) was measured as the time between wounding of the rabbit ear and the cessation of the bleeding, as previously described $[10,11]$.

Blood-citrate tubes were taken and centrifuged at $225 \mathrm{~g} / \mathrm{min}$ for 20 minutes at $25^{\circ} \mathrm{C}$ to recover platelet-rich plasma and further centrifuged at $2000 \mathrm{~g} / \mathrm{min}$ for 10 minutes at $25^{\circ} \mathrm{C}$ to recover platelet-poor plasma. The platelet-rich plasma sample was adjusted at 200 to $25010^{6}$ platelets $/ \mathrm{mL}$ with homologous platelet-poor plasma. Platelets were stimulated with $50 \mathrm{nM} \gamma$-thrombin (a generous gift of $\mathrm{M}$. Jeandrot-Perrus and MC. Guillin), and the aggregation was assessed with a platelet aggrometer (Regulest, Florange, France). $\gamma$-thrombin (a product of limited proteolysis of $\alpha$-thrombin) is not able to cleave fibinogen in fibrin, like $\alpha$ thrombin, but induce platelet aggregation. It was used as an agonist since LMWF have an anti-thrombin activity. Aggregation was expressed as the maximum percent change in light transmittance from baseline, with platelet-poor plasma as a reference.

\subsection{Measurements of plasma tissue factor pathway inhibitor (TFPI) activity.}

TFPI activity was measured in each animal at baseline, and 1 hour after the second subcutaneous injection. TFPI activity in rabbit plasma was determined by a two-step colorimetric assay, as previously described with following modifications [12]. All reagents was obtained from American Diagnostica (Greenwich, CT). Briefly, in the first step, a $1 / 40$ dilution of test sample was incubated with human factor VIla and $20 \mathrm{ng} / \mathrm{mL}$ of rabbit recombinant tissue factor (rTF) complex. In the second step, a saturating concentration of human factor $X$ was added to the reaction mixture as a substrate for the residual factor VIla/TF catalytic activity; the generated factor Xa was measured with the specific chromogenic substrate, Spectrozyme Xa. The resulting absorbance was read at $405 \mathrm{~nm}$. Linear calibration curves were obtained with different dilutions of pooled rabbit plasma. Results are expressed as percent of TFPI activity in this pooled rabbit plasma. 


\subsection{Statistical analysis}

All data were analyzed in a blinded fashion. Parametric data are expressed as mean \pm SD. Non parametric data are expressed as median value \pm upper and lower quartiles. Thrombotic and apoptotic scores, endothelial denudation, and TF expression were averaged from two arteries in each animal, and we used the six average values for the comparison among groups. Thrombosis occurrence was evaluated by recording the number of animals in each group with any thrombus (in one or both arteries). Differences in thrombus formation among groups were evaluated using the Fischer's exact test. A two-way ANOVA test or a KruskalWallis test was used to compare parametric and non parametric data among the 3 groups of animals (control, LMWF, and LMWH groups). If a global significant difference was found, a least-squares difference test for multiple comparisons, or a Mann-Whitney test, when appropriate, was used to identify differences between groups. Values were considered statistically different when $\mathrm{P}<0.05$.

\section{Results}

\subsection{Vessel thrombosis}

We first analyzed thrombosis occurrence in the 3 groups. In the control group, thrombosis occurred in 5 of 6 animals (Figures $1 \mathrm{~A}$ and $1 \mathrm{D}$ ). In the LMWH group, thrombosis occurred in 4 of 6 animals (Figures $1 \mathrm{~B}$ and 1D). Interestingly, in the LMWF group, thrombosis occurred only in 1 animal (Figures $1 \mathrm{C}$ and 1D). The differences in thrombosis occurrence between LMWF and control groups were significant $(p=0.03$, Figure 1D). The severity of the thrombotic response was evaluated by a semi quantitative thrombotic score. The thrombotic score was significantly lower in the LMWF group than in the control and the LMWH groups (Figure 1E). Staurosporin treated arteries exhibited large areas of endothelial denudation (Figure $2 \mathrm{~A}-\mathrm{C}$ ), and remaining endothelial cells were mostly apoptotic (Figure $2 \mathrm{E}-\mathrm{G}$ ). Moreover, endothelial denudation and apoptotic score were closely similar in the 3 groups (Figures $2 \mathrm{I}$ and $2 \mathrm{~J}$ ).

\subsection{Anticoagulant activity}

Anticoagulant activities are summarized in Figure 3.

No significant differences were observed among APTT at baseline in the 3 groups (Figure 3A). The APTTs increased similarly 1 hour after the second and the final injections in the LMWF and the LMWF groups (Figure 3A).

No significant differences were observed among TCT at baseline in the 3 groups (Figure 3B). One hour after the second and the final subcutaneous injections, TCT was dramatically increased in the LMWH groups (> $120 \mathrm{~s}$ ) as compared to the control and LMWF groups. In the LMWF group, TCT was not significantly different as compared to the control group one hour after the second and the final subcutaneous injections (Figure 3B).

Finally, no significant differences were observed among anti-Xa activity at baseline in the 3 groups (Figure $3 \mathrm{C})$. One hour after the second and the final subcutaneous injections, anti-Xa activity was significantly increased in the LMWH group as compared to the control $(p<0.0001)$ and the LMWF group $(p<0.0001)$ (Figure $3 \mathrm{C}$ ). In contrast, anti-Xa activities were similar in the control and the LMWF groups (Figure 3C).

No significant difference of platelet count was observed in the 3 groups between baseline and sacrifice. Decreased in hemoglobin was observed in each group, corresponding to blood depletion induced by blood sampling and surgical procedure (data not shown).

\subsection{Bleeding time and platelet aggregation induced by $\gamma$-thrombin}

Bleeding time was significantly increased in the LMWH group (175 $\pm 17 \mathrm{sec})$ as compared to the control (123 $\pm 20 \mathrm{sec}, \mathrm{p}=0.009)$ and the LMWF group $(124 \pm 23 \mathrm{sec}, \mathrm{p}=0.01)$. In contrast, bleeding time was not significantly different between the LMWF and the control groups. No significant difference of platelet aggregation was observed between the control and the LMWF groups $(68.3 \pm 7.3 \%$ vs $63.2 \pm 7.6 \%$, $\mathrm{p}=0.57)$. In contrast, platelet aggregation was altered in the LMWH group as compared to the control $(22.8 \pm$ $22.5 \%$ vs $68.3 \pm 7.3 \% \mathrm{p}=0.0001)$ and the LMWF $(22.8 \pm 22.5 \%$ vs $63.2 \pm 7.6 \%, \mathrm{p}=0.0005)$.

\subsection{Effect of LMWF in TF pathway}

In the control and the LMWH groups, TF was predominantly and intensively detected in the media, but also in remaining endothelial cells and within the thrombus, as shown in Figures 4A and 4B. In contrast, TF was very low in the media in the LMWF group (Figure 4C). Moreover, TF expression was significantly decreased 
in the LMWF group as compared to the control and the LMWH groups (Figures $4 \mathrm{D}$ ). Interobserver variability of semi-quantitative score of FT expression was evaluated by two blinded observer. Concordance was $86 \%$, and Kappa score was 0.82, indicating excellent agreement.

LMWF induced an increase of TFPI activity (Figure 4E) 1 hour after the second injection compared to baseline. No significant difference in TFPI activity was observed in the control and the LMWH groups 1 hour after the second injection (Figure 4E).

\section{Discussion}

\subsection{Antithrombotic effects of LMWF}

LMWHs are widely used in the treatment of acute coronary syndromes. They are preferred to UF heparin because LMWHs have more predictable bioavailability and pharmacokinetics, and they do not require routine monitoring [13]. Antithrombotic activity of UF and LMWHs are limited by their anticoagulant activity, and thus their hemorrhagic effects. In the present study, we evaluate the antithrombotic effect and the anticoagulant activity of LMWF and LMWH in an experimental model of arterial thrombosis by stimulation of endothelial apoptosis. This model reproduces the mechanisms of thrombotic plaque erosion responsible of $30-40 \%$ of arterial thrombosis, particularly in women [8]. Thrombosis occurrence was particularly low in LMWF treated animals, and significantly lower than in control animals. Moreover, the severity of thrombosis was significantly lower in the LMWF group than in the control and the LMWH groups. In the present study, the antithrombotic effect of LMWF may be in part explained by the effect on the tissue factor (TF) pathway. TF is considered as a major contributor of blood thrombogenicity in acute coronary syndromes [14, 15, 16]. Indeed, in our model, apoptotic endothelial cells may become procoagulant and promote thrombin generation by exposing phosphatidylserine and activating TF $[8,14,15]$. Moreover, tissue factor pathway inhibitor (TFPI) regulates the extrinsic blood coagulation pathway by factor Xa-dependent inhibition of the tissue factor-factor VIIa complex, and thus modulates thrombus formation [17]. Endothelium is considered as the principal source of TFPI, and we hypothesized that TFPI was released by non injured endothelial cells (i.e., upstream or downstream the lesion site) [18]. We observed that plasma TFPI activity level was significantly increased in the LMWF group. This result is in accordance with a previous work demonstrating in vitro that a $20 \mathrm{kDa}$ molecular mass fraction of fucoidan induces TFPI release from human endothelial cells culture in a time- and dose-dependent manner [5]. In contrast, we did not find a significant release of TFPI in animals treated by $\mathrm{LMWH}$. Interestingly, Sundaram $\mathrm{M}$ et al. also reported that release of TFPI is not increased in rabbits with enoxaprin [19]. Furthermore, TF expression was significantly decreased in animals treated by LMWF as compared to the control group. Other properties of LMWF could also interfere with long term inhibition of arterial thrombosis. Indeed, LMWF are able to improve vascular tube formation [20], to inhibit smooth muscle cell proliferation [21, 22], and neointimal hyperplasia [23]. Moreover, in a rat model of critical hindlimb ischemia, LMWF promotes therapeutic revascularization induced by FGF-2 [24].

\subsection{Anticoagulant effect and bleeding risk of LMWF}

LMWF exerts its anticoagulant activity by catalazing thrombin inhibition mainly by heparin cofactor II [2]. We confirmed that the anticoagulant activity of LMWF was low since it was not associated with a significant prolongation of the thrombin clotting time or an increase in anti-Xa activity, contrary to LMWH [6]. Interestingly, for the first time, we demonstrated the absence of accumulation of fucoidan with no increased anticoagulant activities after 4 days of treatment. Moreover, bleeding time and $\gamma$-thrombin-induced platelet aggregation were not altered with LMWF. Our results suggest that the efficacy/bleeding risk ratio of LMWF is particularly promising in the treatment of arterial thrombosis.

\subsection{Study limitations}

Our data were obtained in an experimental model of arterial thrombosis by in situ induction of endothelial apoptosis which plays an important role in thrombotic plaque erosion in humans [8, 25]. Caution is needed before extrapolating these results to thrombotic ruptured plaque or more complex atherosclerotic disease occurring in human.

In conclusion, LMWF, a sulfated polysaccharide extracted from brown seaweeds, is effective to prevent arterial thrombosis in this model with a low bleeding risk. LMWF could be a promising new drug for thrombotic diseases. 


\section{References}

Nardella A, Chaubet F, Boisson-Vidal C, Blondin C, Durand P, Josefonvicz J : Anticogulant low molecular weight fucans produced by radical process and ion exchange chromatography of high molecular weight fucans extracted from the brown seaweed ascophyllum nodosum. Carbohydr Res 1996;289:201-8.

Mauray S, Sternberg C, Theveniaux J, Millet J, Sinquin C, Tapon-Bretaudiere J, Fischer AM: Venous antithrombotic and anticoagulant activities of a fucoidan fraction. Thromb Haemost 1995;74:1280-5.

Bajaj MS, Kuppuswamy MN, Saito H, Spitzer SG, Bajaj SP: Cultured normal hepatocytes do not synthesize lipoprotein-associated coagulation inhibitor: evidence that endothelium is the principle site of its synthesis. Proc Natl Acad Sci USA 1990;87:8869-73.

Lupu C, Poulsen E, Roquefeuil S, Westmuckett AD, Kakkar VV, Lupu F: Cellular effects of heparin on the production and release of tissue factor pathway inhibitor in human endothelial cells in culture. Arterioscler Thromb Vasc Biol 1999;19:2251-62.

Giraux JL, Tapon-Bretaudiere J, Matou S, Fischer AM: Fucoidan, as heparin, induces tissue factor pathway inhibitor release from cultured human endothelial cells. Thromb Haemost 1998;80:692-5.

Millet J, Colliec Jouault S, Mauray S, Theveniaux J, Sternberg C, Boisson Vidal C, Fischer AM: Antithrombotic and anticoagulant activities of a low molecular weight fucoidan by the subcutaneous route. Thromb Haemost 1999;81:391-5.

Colliec-Jouault S, Durand P, Fischer AM: Low molecular weight sulphated polysaccharide to obtain a medicine with antithrombotic activity. United States Patent 2004; $\mathrm{n}^{\circ} 6,828,307$.

Durand E, Scoazec A, Lafont A, Al Haj Zen A, Addad F, Mirshahi M, Desnos M, Tedgui A, Mallat Z: In vivo induction of endothelial apoptosis leads to vessel thrombosis and endothelial denudation. Circulation 2004;109:2503-6.

D'Andrea D, Ravera M, Golino P, Rosica A, De Felice M, Ragni M, Cirillo P, Vigorito F, Corcione N, Tommasini P, Gargiulo A, Piro O, Calabro P, Chiariello M: Induction of tissue factor in the arterial wall during recurrent thrombus formation. Arterioscler Thromb Vasc Biol 2003;23:1684-9.

Carter CJ, Kelton JG, Hirsh J: Comparison of haemorrhagic effect of porcine and bovine heparin in the rabbits. Thromb Res 1979;15:581-6.

Doutremepuich C, Toulemonde F, Bousquet F, Bonini F: Comparison of haemorrhagic effect of unfractionated heparin and a low molecular weight heparin fraction (CY 216) in rabbits. Thromb Res 1986;43:691-5.

Sandset PM, Warn-Cramer BJ, Rao VM, Maki SL, Rapaport SI: Depletion of extrinsic pathway inhibitor (EPI) sensitizes rabbits to disseminate intravascular coagulation induced with tissue factor: Evidence supporting a physiologic role for EPI as a natural anticoagulant. Proc Natl Acad Sci USA 1991;88:708-12.

Cohen M: The role of low-molecular-weight heparin in the management of acute coronary syndromes. J Am Coll Cardiol 2003;41(4 Suppl S):55S-61S.

Giesen PL, Rauch U, Bohrmann B, Kling D, Roque M, Fallon JT, Badimon JJ, Himber J, Riederer MA, Nemerson Y: Blood-borne tissue factor: another view of thrombosis. Proc Natl Acad Sci USA 1999;96: 23115 .

Tedgui A, Mallat Z. Apoptosis as a determinant of atherothrombosis. Thromb Haemost 2001;86:420-6.

Moons AH, Levi M, Peters RJ: Tissue factor and coronary artery disease. Cardiovasc Res 2002;53:313-25.

Ragni M, Golino P, Cirillo P, Scognamiglio A, Piro O, Esposito N, Battaglia C, Botticella F, Ponticelli P, Ramunno L, Chiariello M: Endogenous tissue factor pathway inhibitor modulates thrombus formation in an in vivo model of rabbit carotid artery stenosis and endothelial injury. Circulation 2000;102:113-7.

Morange PE, Simon C, Alessi MC, Luc G, Arveiler D, Ferrieres J, Amouyel P, Evans A, Ducimetiere P, Juhan-Vague I; PRIME Study Group: Endothelial cell markers and the risk of coronary heart disease: the Prospective Epidemiological Study of Myocardial Infarction (PRIME) study. Circulation 2004;109:1343-8.

Sundaram M, Qi Y, Shriver Z, Liu D, Zhao G, Venkataraman G, Langer R, Sasisekharan R: Rational design of low-molecular weight heparins with improved in vivo activity. Proc Natl Acad Sci USA 2003;100:651-6.

Chabut D, Fischer AM, Colliec-Jouault S, Laurendeau I, Matou S, Le Bonniec B, Helley D: Low molecular weight fucoidan and heparin enhance the FGF-2 induced tube formation of endothelial cells through heparan sulfate-dependent alpha 6 over-expression. Mol Pharmacol 2003;64:696-702.

Logeart D, Prigent-Richard S, Jozefonvicz J, Letourneur D: Fucans, sulfated polysaccharides extracted from brown seaweeds, inhibit vascular smooth muscle cell proliferation. I. Comparison with heparin for antiproliferative activity, binding and internalization. Eur J Cell Biol 1997;74:376-84.

Logeart D, Prigent-Richard S, Boisson-Vidal C, Chaubet F, Durand P, Jozefonvicz J, Letourneur D: Fucans, sulfated polysaccharides extracted from brown seaweeds, inhibit vascular smooth muscle cell proliferation. II. Degradation and molecular weight effect. Eur J Cell Biol 1997;74:385-90.

Deux JF, Meddahi-Pelle A, Le Blanche AF, Feldman LJ, Colliec-Jouault S, Bree F, Boudghene F, Michel JB, Letourneur D: Low molecular weight fucoidan prevents neointimal hyperplasia in rabbit iliac artery in-stent restenosis model. Arterioscler Thromb Vasc Biol 2002;22:1604-9. 
Luyt CE, Meddahi-Pelle A, Ho-Tin-Noe B, Colliec-Jouault S, Guezennec J, Louedec L, Prats H, Jacob MP, Osborne-Pellegrin M, Letourneur D, Michel JB: Low-molecular-weight fucoidan promotes therapeutic revascularization in a rat model of critical hindlimb ischemia. J Pharmacol Exp Ther 2003;305:24-30.

Virmani R, Kolodgie FD, Burke AP, Farb A, Schwartz SM. Lessons from sudden coronary death: a comprehensive morphological classification scheme for athero-sclerotic lesions. Arterioscler Thromb Vasc Biol 2000;20:1262-75.

\section{Figures}
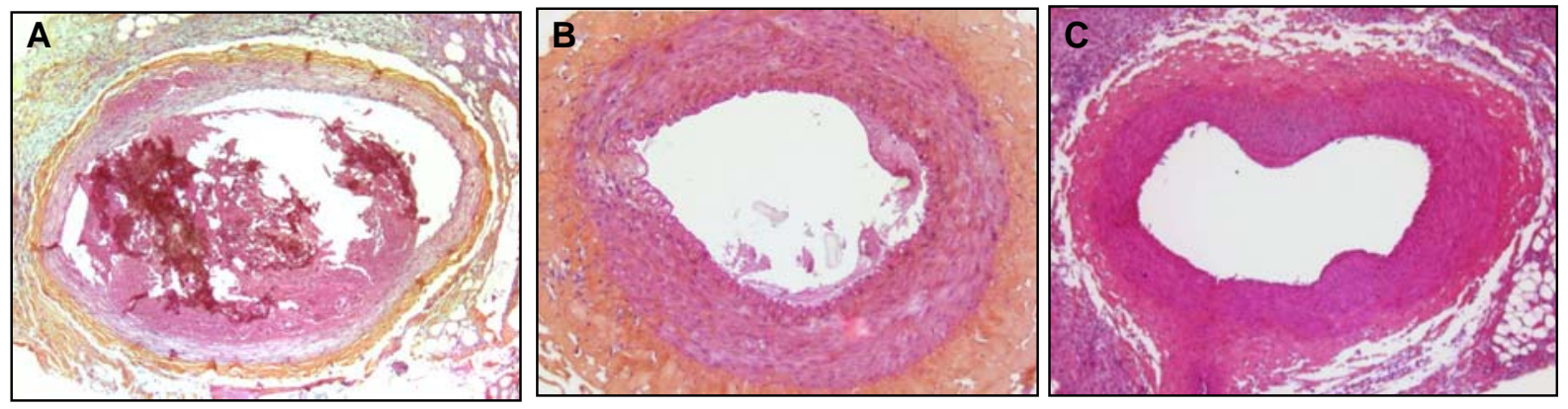

D
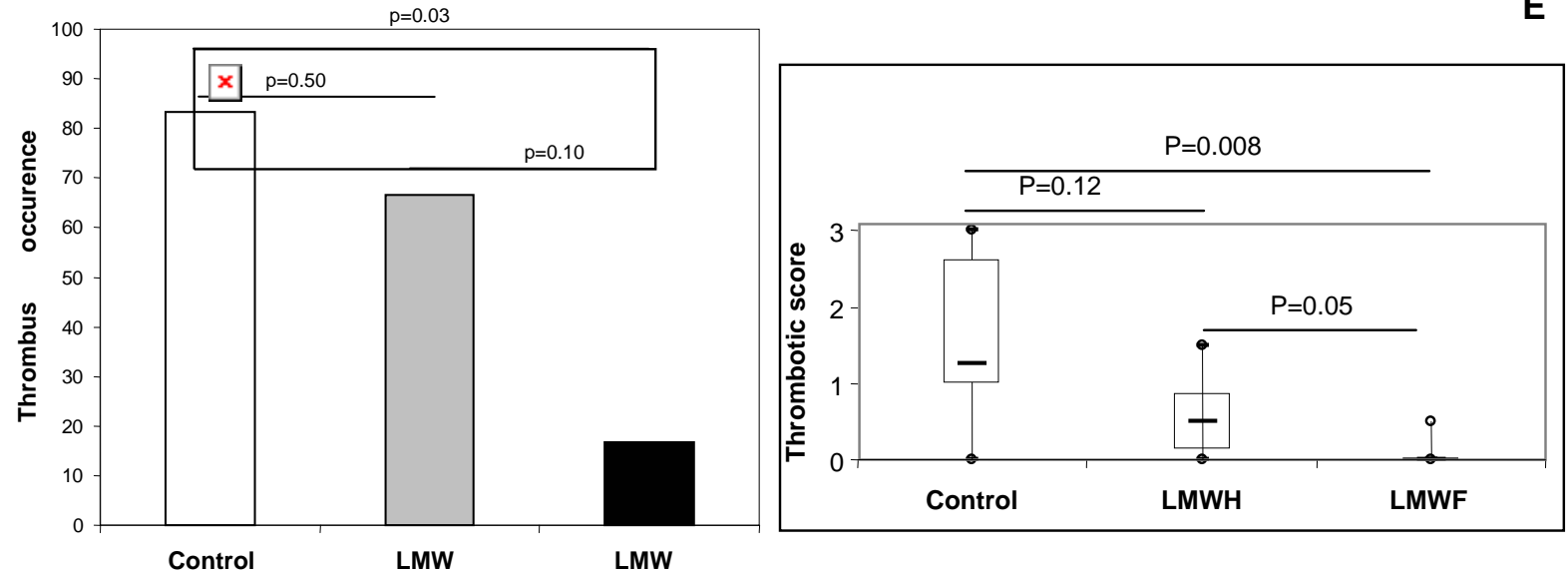

Figure 1. Antithrombotic effects LMWF.

Thrombosis occurrence was evaluated 3 days after in vivo induction of endothelial apoptosis in control (A), LMWH (B), and LMWF (C) groups. D : Bar graph representation of thrombosis occurrence in control (white), LMWH (grey), and LMW fuocoidan (black) treated animals. $\mathrm{N}=6$ in each group. Fischer's exact test was used to compare thrombosis occurrence between groups. E: Turkey box plot of the severity of thrombus (thrombotic score) in control, LMWH, and LMWF (groups). Horizontal bar represent median value, and points indicates $90^{\text {th }}$ and $10^{\text {th }}$ percentiles Original Magnification: X5 (A-C). Staining: hematoxylin and eosin (A-C). 

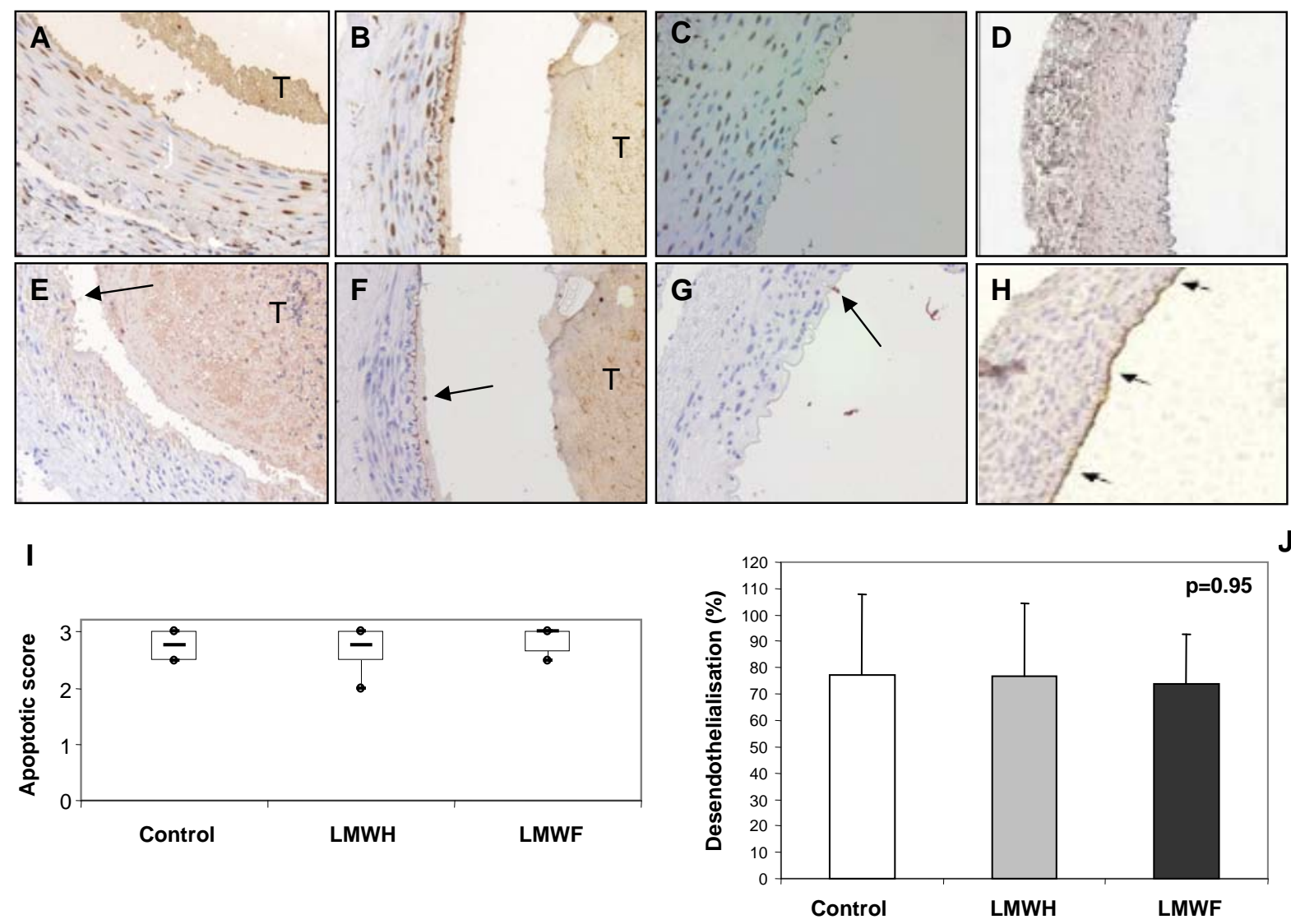

Figure 2. Induction of endothelial apoptosis and endothelial denudation.

Endothelial apoptosis and denudation were evaluated by immunohistochemistry 3 days after in vivo incubation with staurosporin in control (A, E), LMWH (B, F) and LMWF (C, G) groups. Apoptotic endothelial cells were detected by TUNEL (A-C) and endothelial denudation was evaluated by anti-CD31 antibody (E$G)$. Apoptotic cells $(A-C)$, and remaining endothelial cells (D-F) appeared with a brown nuclei (arrows, A-C). Non apoptotic cells had purple nuclei (A-C). Negative control for TUNEL staining (D), and positive control for anti CD-31 staining (arrows, H) in normal femoral rabbit artery. Original Magnification: X20. T: indicating thrombus (A, B, E, F). I: Turkey box plot of the severity of apoptosis (apoptotic score) in control, LMWH, and LMWF groups. Horizontal bar represent median value, and points indicates $90^{\text {th }}$ and $10^{\text {th }}$ percentiles. J: Bar graph representation of endothelial denudation (F), in control (white), LMWH (grey), and LMWF (black) treated animals. Endothelial denudation and apoptosis were similar in the 3 groups $(p=0.95, p=0.97$; respectively). 
$\square$ Contro

$\square$ LMW

- LMWF

A

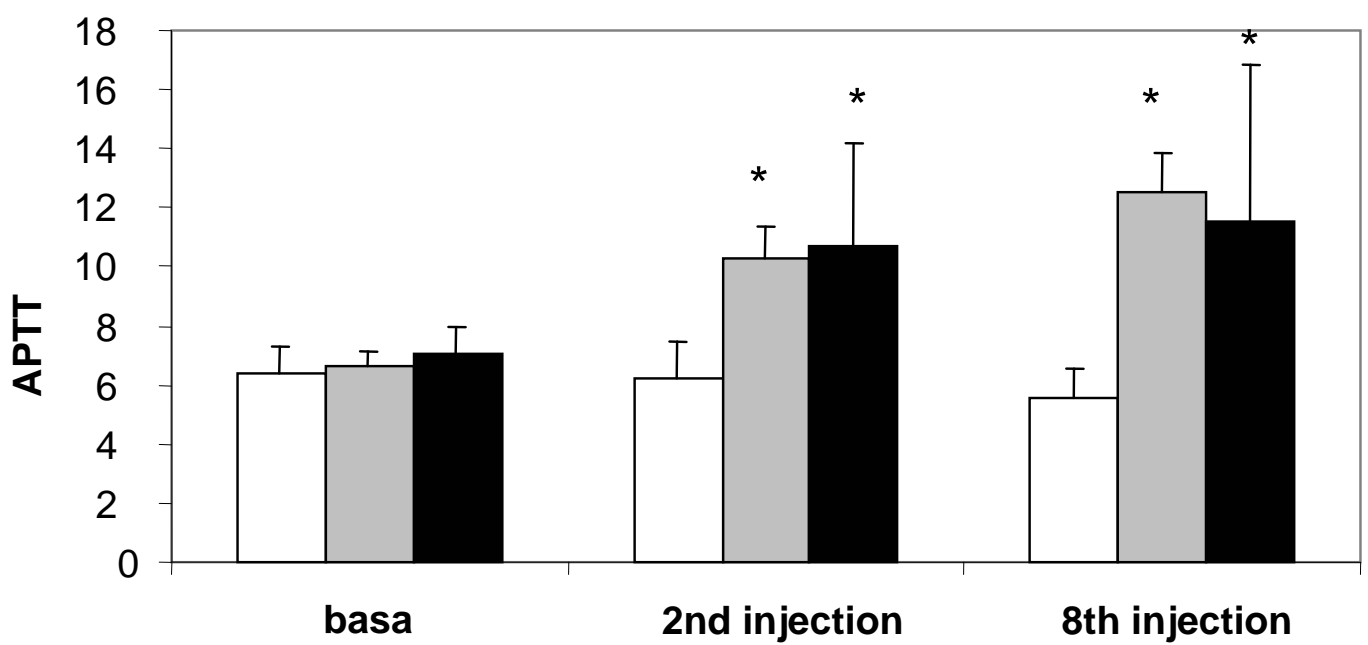

B
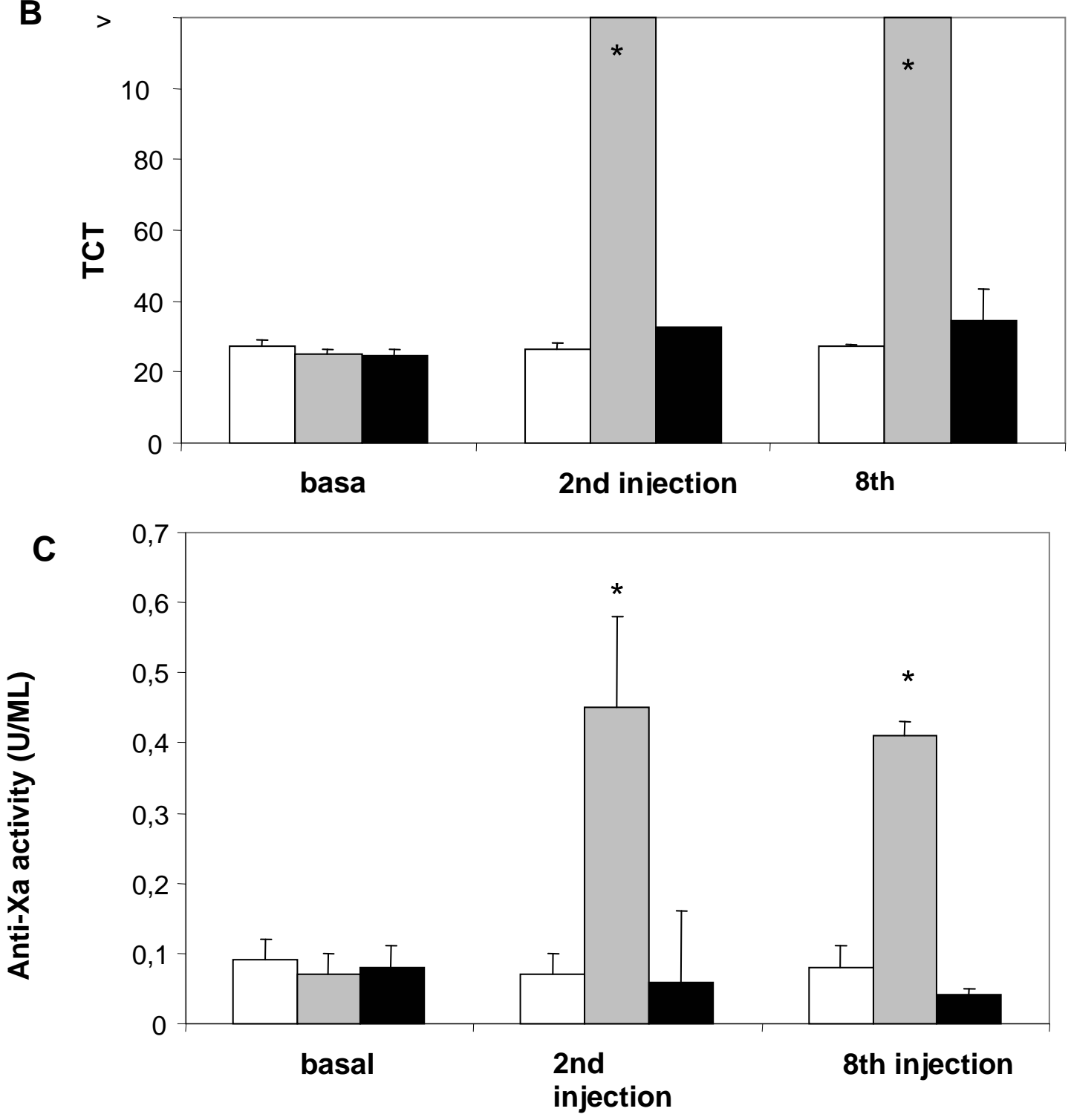
Figure 3. Anticoagulant activity of LMWF

Bar graph representation of APTT (A), TCT (B) and anti-Xa activity (C) at baseline, 1 hour after the second subcutaneous administration, and 1 hour after the final $\left(8^{\text {th }}\right)$ injection in animals treated by a saline solution (control, white), LMWH (grey), and LMWF (black). * $\mathrm{P}<0.05$.
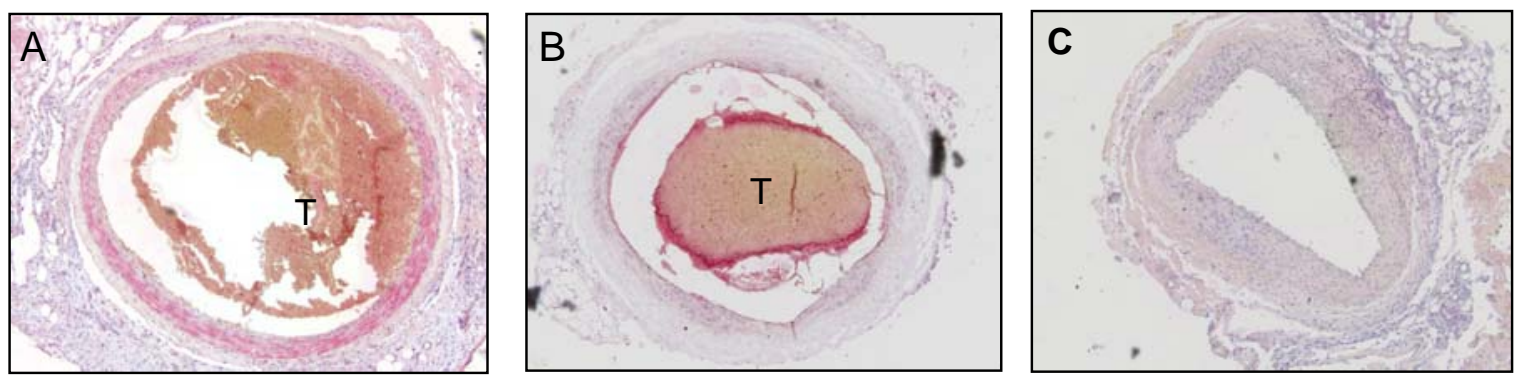

D

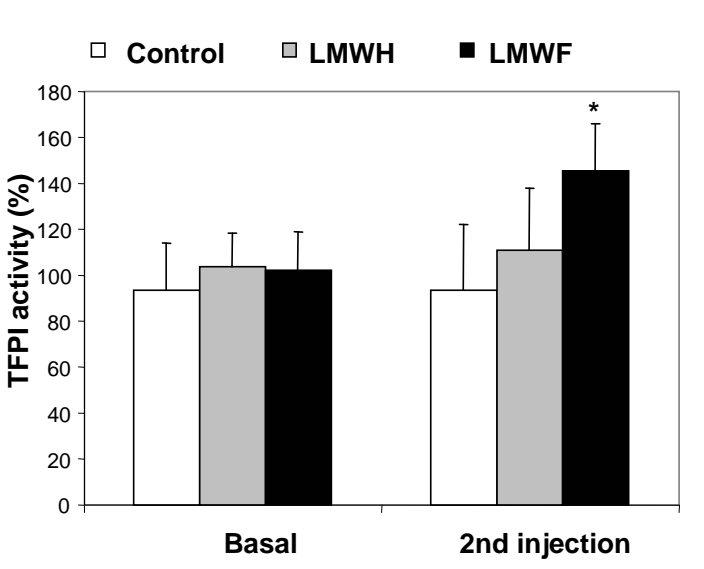

Figure 4. Effect on tissue factor pathway

TF expression was evaluated 3 days after in vivo induction of endothelial apoptosis by an anti-rabbit tissue factor monoclonal antibody in control (A), LMWH (B), and LMWF (C) treated animals. Cells which expressed TF were stained red. TF expression was detected in the control and the LMWH groups in remaining endothelial cells and strongly in the media (A, B). In contrast, TF expression was barely detectable in the LMWF group (C). Original Magnification: X5 (A-C). T: indicating thrombus (A, B). D: Turkey box plot of TF expression in control, LMWH, and LMWF groups. Horizontal bar represent median value, and points indicates $90^{\text {th }}$ and $10^{\text {th }}$ percentiles * $P<0.05$. E: Bar graph representation of plasma TFPI activity at baseline, and 1 hour after the second subcutaneous administration in animals treated by a saline solution (control, white), LMWH (grey), and LMWF (black). For TFPI activity, 100\% corresponded to the value obtained in rabbit pooled plasma. $\mathrm{N}=6$ in each group. ${ }^{*} \mathrm{P}<0.05$. 